\title{
Long-term safety and efficacy of guanfacine extended release in children and adolescents with ADHD
}

\author{
Michael Huss ${ }^{1} \cdot$ Bryan Dirks $^{2,3} \cdot$ Joan Gu$^{4} \cdot$ Brigitte Robertson $^{4} \cdot$ Jeffrey H. Newcorn ${ }^{5} \cdot$ J. Antoni Ramos-Quiroga ${ }^{6}$
}

Received: 19 July 2017 / Accepted: 15 January 2018 / Published online: 13 February 2018

(c) The Author(s) 2018. This article is an open access publication

\begin{abstract}
Data are reported from SPD503-318, a phase 3, open-label, safety study of guanfacine extended release (GXR) in European children and adolescents with attention-deficit/hyperactivity disorder (ADHD). Participants received dose-optimized GXR (1-7 mg/day) for up to 2 years. Of 215 enrolled participants, 214 were included in the safety population and 133 completed the study. Participants' mean age was 11.7 years and $73.8 \%$ were male. Overall, 177 participants (82.7\%) experienced a treatment-emergent adverse event (TEAE). TEAEs reported in at least $10 \%$ of participants were somnolence $(36.0 \%)$, headache $(28.5 \%)$, fatigue $(20.1 \%)$, and nasopharyngitis (11.7\%). Serious TEAEs were reported in $4.7 \%$ of participants and TEAEs leading to discontinuation were reported in 3.3\% of participants. There were no deaths. Mean $z$-scores for BMI were stable throughout the study. The incidence of sedative TEAEs (somnolence, sedation, and hypersomnia) peaked during week 3 and decreased thereafter. Small changes from baseline to the final assessment in mean supine pulse [ $-5.5 \mathrm{bpm}$ (standard deviation, 12.98)] and blood pressure [systolic, $0.6 \mathrm{mmHg}$ (9.32); diastolic, $0.2 \mathrm{mmHg}$ (9.17)] were reported. ADHD symptoms initially decreased and remained significantly lower than baseline at study endpoint. At the final assessment, the mean change in ADHD-RS-IV total score from baseline was - 19.8 (standard error of mean, 0.84; nominal $p<0.0001$ ). In conclusion, GXR was well tolerated and more than $60 \%$ of participants completed the 2 -year study.
\end{abstract}

Keywords Attention-deficit/hyperactivity disorder $\cdot$ Child/adolescent $\cdot$ Clinical trial $\cdot$ Guanfacine extended release . Efficacy $\cdot$ Safety

Electronic supplementary material The online version of this article (https://doi.org/10.1007/s00787-018-1113-4) contains supplementary material, which is available to authorized users.

Michael Huss

m.huss@rfk.landeskrankenhaus.de

1 Child and Adolescent Psychiatry, Johannes Gutenberg University Mainz, Mainz, Germany

2 Shire, Wayne, PA, USA

3 Present Address: ICON Clinical Research, North Wales, PA, USA

4 Shire, Lexington, MA, USA

5 Department of Psychiatry, Icahn School of Medicine at Mount Sinai, New York, NY, USA

6 Department of Psychiatry (CIBERSAM), Hospital Universitari Vall d'Hebron and Universitat Autònoma de Barcelona, Barcelona, Spain

\section{Introduction}

Attention-deficit/hyperactivity disorder (ADHD) is a chronic disorder that is estimated to affect $5.3 \%$ of children worldwide $[1,2]$. Symptoms persist into adulthood in up to $66 \%$ of children diagnosed with ADHD, causing lifelong impairment $[3,4]$. Pharmacotherapy has been shown to reduce ADHD symptoms, and reports also suggest that it can improve outcomes such as academic performance, self-esteem, and social functioning [5]. According to practice guidelines, drug treatment for ADHD should be continued for as long as it remains clinically effective and should be monitored regularly $[6,7]$. Results from studies in Canada and the UK have shown that up to $80 \%$ of individuals persisted with their ADHD medication for 1 year and $60 \%$ remained on treatment after 2 years $[8$, 9]. Although adherence in clinical practice is highly variable, the duration of drug exposure in real life is likely to be longer than that typically assessed in registrational trials. In particular, concerns have been raised about potential long-term effects of 
stimulant and non-stimulant ADHD medications on growth and weight [10-13].

GXR is a non-stimulant treatment for patients with ADHD. In the USA and Canada, GXR is approved for use in children and adolescents as monotherapy or adjunctive to stimulant therapy [14]. In Europe, GXR is approved for the treatment of children and adolescents (aged 6-17 years) with ADHD when stimulants are unsuitable, not tolerated, or have been shown to be ineffective [12]. The safety and efficacy of GXR in children and adolescents with ADHD have been established in short-term trials of GXR. SPD503-301 and SPD503-304 were 5-6 week, forced-dose, phase 3 studies of GXR doses of up to $4 \mathrm{mg} /$ day in children and adolescents $[15,16]$. SPD503312 (adolescents) and SPD503-316 (children and adolescents) were 10-13-week, flexible-dose, phase 3 studies of GXR doses of up to $7 \mathrm{mg} /$ day $[17,18]$. The need for ongoing GXR treatment was demonstrated in SPD503-315, a 39-week doubleblind, randomized, placebo-controlled withdrawal study of participants who had responded to an initial 13-week openlabel GXR treatment phase. In this study, treatment failure occurred in $64.9 \%$ of participants withdrawn to placebo compared with $49.3 \%$ of those who were maintained on GXR $(p=0.006)$ [19]. Long-term safety and efficacy data for GXR doses of up to $4 \mathrm{mg} /$ day are available from 2-year studies in children and adolescents with ADHD in the USA (SPD503303 and SPD503-305) [20, 21].

In all of the above studies, sedative events were the most common adverse events, which typically emerged within the first 2 weeks of treatment and generally resolved thereafter. Modest mean decreases in pulse rate were observed with GXR treatment in all studies, as well as decreases in mean blood pressure in all but SPD503-305 (which included combination therapy with psychostimulants). Long-term GXR treatment was not associated with abnormal changes in weight or height at the group level, but in some individuals, weight increases were observed. These findings are reflected in the European Medicines Agency's risk management plan for GXR, which identifies sedative events, low blood pressure, and weight increase as important risks [22].

The aim of the present study was to evaluate the longterm safety and tolerability of GXR administered at doses of up to $7 \mathrm{mg} /$ day in children and adolescents with ADHD. Results are reported from SPD503-318, (ClinicalTrials.gov identifier: NCT01500694) with a focus on sedation, vital signs, and weight.

\section{Methods}

\section{Ethics and conduct}

SPD503-318 was conducted in accordance with the International Conference on Harmonisation Guidelines for Good
Clinical Practice (GCP), the principles of the Declaration of Helsinki, and other applicable local ethical and legal requirements. Informed consent from the participants, or informed assent plus consent from legally authorized representatives, was obtained before entry into the study. The study took place between 20 March 2012 and 15 September 2015 at 52 sites in 11 European countries (Austria, Belgium, France, Germany, Italy, The Netherlands, Poland, Romania, Spain, the UK, and Ukraine).

\section{Participants}

SPD503-318 was a phase 3, open-label, single-arm extension study of European participants in the international GXR phase 3 studies SPD503-316 and SPD503-315 (ClinicalTrials.gov identifiers: NCT01244490 and NCT01081145). Both antecedent studies enrolled children (aged 6-12 years) and adolescents (aged 13-17 years) with an Attention-Deficit/ Hyperactivity Disorder Rating Scale IV (ADHD-RS-IV) total score of at least 32 and a Clinical Global Impressions-Severity of Illness (CGI-S) score of at least 4 at baseline [17, 19]. Participants in SPD503-316 were randomized to receive GXR, atomoxetine or placebo, and participants in SPD505-315 were randomized to receive either GXR or placebo. To be eligible for inclusion in SPD503-318, participants in SPD503-316 had to have completed the double-blind evaluation, reached the final visit, completed the 2-week dose-tapering period, and attended the safety follow-up visit. Participants in SPD503-315 were eligible for inclusion in SPD503-318 if they met the response criteria for entry into the double-blind, randomized-withdrawal period, completed the randomized-withdrawal period (or withdrew early) and completed a 2-week dose-tapering period and attended a safety follow-up visit. Additional inclusion criteria for SPD503-318 were supine and standing blood pressure measurements below the 95th percentile for age, sex, and height.

Key exclusion criteria for SPD503-318 were: any current co-morbid psychiatric diagnosis (excluding oppositional defiant disorder [ODD]); a history of alcohol or other substance abuse or dependence; a body mass index (BMI) at screening above the 95 th percentile (using the 2000 Centers for Disease Control and Prevention [CDC] BMI-for-age sexspecific charts [23]); a body weight at screening of less than $25 \mathrm{~kg}$ if aged 6-12 years, or less than $34 \mathrm{~kg}$ if aged 13 years or older; a risk of suicide (in the opinion of the investigator), a previous suicide attempt or a history of/current active suicidal ideation (participants with intermittent passive suicidal ideation were not necessarily excluded, based on the assessment of the investigator); the presence of clinically significant electrocardiogram (ECG) findings, as judged by the investigator; a history or presence of structural cardiac abnormalities, serious heart rhythm abnormalities, syncope, 
cardiac conduction problems, exercise-related cardiac events including syncope and pre-syncope, or clinically significant bradycardia; and orthostatic hypotension or a known history of hypertension.

\section{Study design}

The overall duration of open-label treatment in SPD503318 was up to 104 weeks, comprising up to 7 weeks of dose optimization, 95 weeks of dose maintenance, and 2 weeks of dose tapering (Supplementary Fig. 1). Before the baseline visit, participants were required to undergo a 30-day (SPD503-316) or 7-day (SPD503-315) drug washout period.

GXR was initiated at $1 \mathrm{mg} /$ day, and in the absence of any significant safety issues, the daily dose could be increased or decreased weekly by $1 \mathrm{mg}$ until an optimal dose was reached, up to the maximum permitted dose for the participant's age group (4 mg/day for children and 4-7 mg/day for adolescents, dependent on body weight). The optimal dose was determined by the investigator, taking into account parental and participant's reports, ADHD-RS-IV and CGI-S scale scores, and the safety and tolerability profile of the study drug for each participant. Dose adjustments of $1 \mathrm{mg} /$ day were permitted at any time based on clinical judgement of tolerability and efficacy.

\section{Safety}

The primary objective of this study was to evaluate the long-term safety and tolerability of GXR based on treatment-emergent adverse events (TEAEs), vital signs, ECG parameters, and growth. TEAEs were assessed at each visit and coded using the Medical Dictionary for Regulatory Activities (MedDRA) version 12.1. Participants were asked a non-leading general question to ascertain whether any TEAEs had occurred since the previous visit. Any symptoms reported during the assessment visits that were deemed to be clinically significant by the investigator were also recorded. In SPD503-318, TEAEs related to somnolence, sedation, and hypersomnia were grouped as sedative TEAEs. TEAEs pre-defined as being of special interest were aggression and sedative TEAEs. Any new onset of seizures, loss of consciousness, or syncope was to be recorded as a serious TEAE. Vital signs included pulse rate, systolic blood pressure (SBP) and diastolic blood pressure (DBP) and were also assessed at each visit. ECG parameters included heart rate and QT interval corrected using Fridericia's formula $(\mathrm{QTcF})$, and were assessed at baseline, visits 10, 15, and 19, and follow-up.

Potentially clinically important (PCI) thresholds were pre-defined based on clinical experience for the following measures: supine pulse rate $(\leq 50 ; \geq 100 \mathrm{bpm})$, supine SBP ( $<90 \mathrm{mmHg}$ [children] or $<100 \mathrm{mmHg}$ [adolescents];
$>120 \mathrm{mmHg}$ [children] or $>140 \mathrm{mmHg}$ [adolescents]), supine DBP $(<50 \mathrm{mmHg}$ [children] or $<60 \mathrm{mmHg}$ [adolescents]; $>80 \mathrm{mmHg}$ [children] or $>90 \mathrm{mmHg}$ [adolescents]), resting heart rate based on ECG $(\leq 50 ; \geq 100 \mathrm{bpm})$, and QTcF interval ( $\geq 500 \mathrm{~ms}$ ).

Height and weight were assessed at baseline, visit 9, and visits 11-21. BMI was calculated as weight $/$ height $^{2}\left(\mathrm{~kg} / \mathrm{m}^{2}\right)$. Weight was categorized as: underweight, $<5$ th percentile; healthy weight, $\geq 5$ th to $<85$ th percentiles; overweight, $\geq 85$ th to $<95$ th percentiles; and obese, $\geq 95$ th percentile. To derive $z$-scores adjusted for age (at assessment), height, weight, and BMI measurements were compared with the 2000 CDC growth charts for children and adolescents aged 2-20 years [23]. Possible emergent suicidal ideation and behaviour were assessed at each visit using the ColumbiaSuicide Severity Rating Scale (C-SSRS).

\section{Efficacy}

The secondary objective of this study was to assess ADHD symptoms over 2 years of GXR treatment based on ADHDRS-IV total score and Hyperactivity/Impulsivity and Inattention subscale scores, and the CGI-S scale (all were assessed at baseline and visits 3-19) [24, 25].

\section{Data analysis}

Safety analyses were based on reports obtained in the safety population, defined as all enrolled participants who received at least one dose of GXR. Efficacy analyses were based on ratings of ADHD symptoms in the full analysis set (FAS), which was defined as all enrolled participants who received at least one dose of GXR, excluding participants from one site where a breach of GCP regulations was reported.

The sample size was not determined by statistical considerations because this was a single-arm open-label study with no a priori hypotheses. Missing data were handled using the last observation carried forward method. Changes from baseline to the final assessment in ADHD-RS-IV scores were assessed using a one-sample $t$ test; $p$ values (nominal, unadjusted for multiplicity) were calculated for descriptive purposes.

\section{Results}

\section{Study population}

The disposition of the study population is shown in Fig. 1. Of 215 participants who were enrolled in the study, 214 received at least one dose of GXR and were included in the safety population. Of these, 138 participants were enrolled from SPD503-316 (45 randomized to receive GXR and 48 to 
receive atomoxetine and 45 to receive placebo) and 76 participants were enrolled from SPD503-315 (43 randomized to receive GXR and 33 to receive placebo).

The FAS excluded five participants from one site because of a breach of GCP regulations and, therefore, comprised 209 participants. In total, 133 participants $(62.1 \%)$ completed the study. Among the 81 participants who did not complete the study, the most common reason for early termination was 'withdrawal by participant' $(17.3 \%, n=37)$. Early termination due to lack of efficacy was reported by 19 participants (8.9\%).

\section{Baseline characteristics}

Most participants were male $(158 / 214 ; 73.8 \%)$ and the mean age of the study population was 11.7 years (standard deviation [SD], 2.82) (Table 1). At baseline, the mean ADHD-RSIV total score was 36.7 (SD, 10.41) and 21/214 participants (9.8\%) had a diagnosis of ODD. Prior to the antecedent studies, approximately half of the participants $(111 / 214 ; 51.9 \%)$ had a history of the previous stimulant ADHD medication and about one-third $(62 / 214 ; 29.0 \%)$ had a history of nonstimulant ADHD medication.
Fig. 1 Summary of participant disposition. ${ }^{a}$ In SPD503-315, 43 participants received GXR and 33 received placebo. ${ }^{\mathrm{b}}$ In SPD503-316, 45 participants received GXR, 48 received ATX, and 45 received placebo. ${ }^{\mathrm{c}}$ All enrolled participants who took at least one dose of GXR during the study. ${ }^{\mathrm{d}}$ The FAS excluded five participants enrolled at site 403 , where a breach of Good Clinical Practice regulations was identified. ${ }^{\mathrm{e}}$ All enrolled participants who received at least one dose of GXR, excluding participants from site 403. ATX atomoxetine, $F A S$ full analysis set, $G X R$ guanfacine extended release

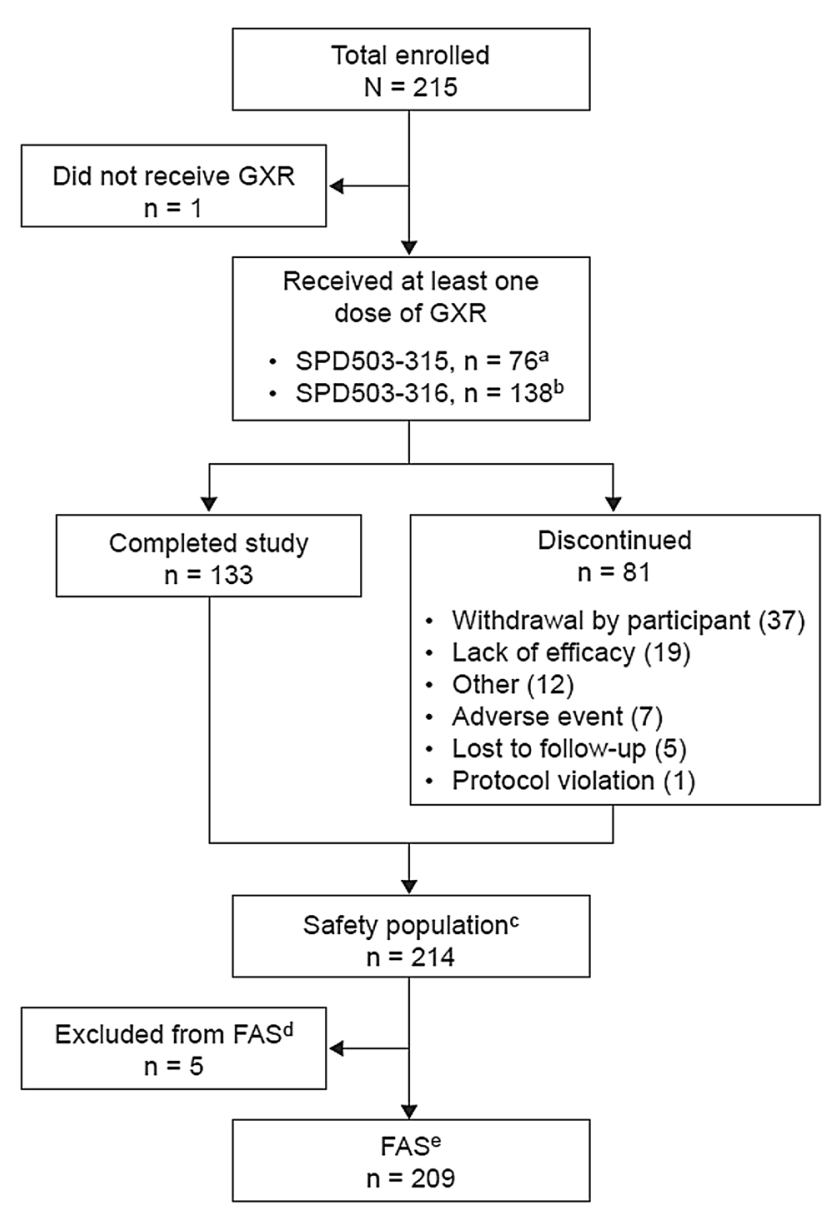

${ }^{a}$ In SPD503-315, 43 participants received GXR and 33 received placebo.

${ }^{\mathrm{b}}$ In SPD503-316, 45 participants received GXR, 48 received ATX, and 45 received placebo.

${ }^{\mathrm{c}}$ All enrolled participants who took at least one dose of GXR during the study.

${ }^{\mathrm{d}}$ The FAS excluded 5 participants enrolled at site 403 where a breach of Good Clinical Practice regulations was identified.

${ }^{\mathrm{e}}$ All enrolled participants who received at least one dose of GXR, excluding participants from site 403.

ATX, atomoxetine; FAS, full analysis set; GXR, guanfacine extended release. 
Table 1 Baseline demographics and disease characteristics (safety population, $n=214$ )

\begin{tabular}{ll}
\hline Characteristic & \\
\hline Demographics & \\
Age, years, mean (SD) & $11.7(2.82)$ \\
$6-12, n(\%)$ & $131(61.2)$ \\
$13-18, n(\%)$ & $83(38.8)$ \\
Sex, male, $n(\%)$ & $158(73.8)$ \\
Race, white, $n(\%)^{\mathrm{a}}$ & $204(95.3)$ \\
Height, cm, mean (SD) & $151.45(15.709)$ \\
Weight, kg, mean (SD) & $45.53(14.505)$ \\
BMI, kg/m ${ }^{2}$, mean (SD) & \\
Time since ADHD diagnosis, years, mean (SD) & $3.1(2.94)$ \\
Disease characteristics & \\
ADHD-RS-IV total score, mean (SD) & $36.7(10.41)$ \\
ADHD subtype, $\mathrm{n}(\%)$ & \\
Combined & $178(83.2)$ \\
Predominantly Inattentive & $20(9.3)$ \\
Predominantly Hyperactive/Impulsive & $16(7.5)$ \\
Current psychiatric comorbidities, $\mathrm{n}(\%)$ & \\
ODD & $21(9.8)$ \\
Other & $1(0.5)$ \\
None & $192(89.7)$ \\
\hline
\end{tabular}

${ }^{a}$ Owing to privacy laws, data regarding race were not permitted to be captured in France

${ }^{\mathrm{b}}$ Calculated at screening

$A D H D$ attention-deficit/hyperactivity disorder, $A D H D-R S-I V$ ADHD Rating Scale IV, BMI body mass index, $O D D$ oppositional defiant disorder, $S D$ standard deviation

\section{Guanfacine extended release exposure}

The median daily dose of GXR during the active treatment period (i.e., before tapering) was $4 \mathrm{mg}$ (mean, $3.6 \mathrm{mg}$; SD, 1.30), with 115/214 (53.7\%) receiving GXR 4-7 mg/day. Of these, 57 were children and 58 were adolescents. At the final assessment, weight-adjusted doses of GXR were within the recommended range of $0.05-0.12 \mathrm{mg} / \mathrm{kg}$ in $176 / 214$ participants $(82.2 \%)$, including 50/214 (23.4\%) who received GXR in the higher range of $0.09-0.12 \mathrm{mg} / \mathrm{kg}$. Of the participants who did not receive GXR within the recommended range, $4 / 214(1.9 \%)$ received a higher dose of $0.13-0.16 \mathrm{mg} / \mathrm{kg}$. The mean length of exposure to GXR in the present study was 564.5 days; when combined with the duration of antecedent studies, the mean length of exposure was 649.2 days.

\section{Treatment-emergent adverse events}

No deaths occurred during the study. In total, 933 TEAEs were reported in $177 / 214$ participants $(82.7 \%)$ and fewer than $10 \%$ of the TEAEs were severe (Table 2). TEAEs reported in more than $10 \%$ of participants were somnolence, headache, fatigue and nasopharyngitis (Table 2) and there were no obvious associations with weight-adjusted dose (Supplementary Table 1).

Ten participants $(4.7 \%)$ had a total of 13 serious TEAEs (aggression, appendicitis, concussion, gastroenteritis, limb fractures, post-procedural haemorrhage, stomatitis and testicular torsion). Serious TEAEs that occurred in more than one participant were limb fractures (four events; reported as lower limb [moderate], radius [severe], upper limb [mild] and wrist [severe]) and concussions (two events). None of the serious TEAEs of fractures occurred after a reported sedative event, dizziness or light-headedness. All serious TEAEs were considered to be unrelated to GXR and resolved during the study; none led to early termination. No TEAEs of syncope were reported.

TEAEs that led to early termination (seven participants, $3.3 \%$ ) were somnolence (two participants), aggression, firstdegree atrioventricular block, dizziness, drug abuse and increased weight. All of these, except drug abuse, were considered by the investigator to be related to GXR and resolved either during dose tapering or after GXR discontinuation. The drug abuse TEAE was a moderate event of cannabis use that lasted for approximately 7 months from the baseline visit. The somnolence and aggression TEAEs that led to early termination were reported as severe.

\section{TEAEs of special interest}

Overall, 125 sedative TEAEs were reported in 81 participants; most events were mild or moderate in severity (Table 2). Of the three types of sedative events, somnolence was more common than sedation and hypersomnia. Sedative TEAEs had a median onset of 21 days after initiation of GXR therapy, and a median duration of 11.0 days. Over the course of the study, the incidence and prevalence of sedative TEAEs were highest during weeks 3 and 4 of dose optimization and then decreased during the dose-maintenance period (Fig. 2). Two participants withdrew from the study because of sedative TEAEs; both experienced events of severe somnolence that were considered by the investigator to be related to GXR. One event started on study day 1 , led to withdrawal after 7 days and resolved on the day of GXR discontinuation (dose at onset, $1 \mathrm{mg} /$ day). The other event led to study withdrawal on day 49 (6 days after onset) and resolved during dose tapering (total duration, 12 days; dose at onset, $4 \mathrm{mg} / \mathrm{day})$.

Nine participants $(4.2 \%)$ reported ten TEAEs of aggression. Five aggression events started during the first 8 weeks of treatment and the other five events began between months 6 and 11. Four aggression TEAEs were considered by the investigator to be related to GXR, but the one serious aggression TEAE was considered to be unrelated to GXR. Including the serious event, nine out of the 10 events 
Table 2 Treatment-emergent adverse events (safety population, $n=214$ )

\begin{tabular}{|c|c|c|}
\hline & Participants, $n(\%)$ & Events, $n$ \\
\hline \multicolumn{3}{|l|}{ TEAE } \\
\hline Any TEAE & $177(82.7)$ & 933 \\
\hline TEAEs related to GXR & $132(61.7)$ & 328 \\
\hline TEAEs leading to dose reduction & $57(26.6)$ & 92 \\
\hline TEAEs leading to early termination & $7(3.3)$ & $7^{\mathrm{a}}$ \\
\hline Severe TEAE & $19(8.9)$ & 23 \\
\hline Serious TEAEs & $10(4.7)$ & $13^{\mathrm{b}}$ \\
\hline TEAEs leading to death & 0 & 0 \\
\hline \multicolumn{3}{|l|}{ TEAEs reported in $\geq 5 \%$ of participants } \\
\hline Somnolence & $77(36.0)$ & 119 \\
\hline Headache & $61(28.5)$ & 134 \\
\hline Fatigue & $43(20.1)$ & 58 \\
\hline Nasopharyngitis & $25(11.7)$ & 38 \\
\hline Dizziness & $21(9.8)$ & 26 \\
\hline Insomnia & $16(7.5)$ & 17 \\
\hline Nausea & $14(6.5)$ & 17 \\
\hline Upper respiratory tract infection & $13(6.1)$ & 26 \\
\hline Abdominal pain & $13(6.1)$ & 15 \\
\hline Vomiting & $11(5.1)$ & 16 \\
\hline Rhinitis & $11(5.1)$ & 14 \\
\hline Oropharyngeal pain & $11(5.1)$ & 13 \\
\hline \multicolumn{3}{|l|}{ Sedative TEAEs, $n=125^{\mathrm{c}}$} \\
\hline Somnolence events, $n$ & 119 & \\
\hline Sedation events, $n$ & 5 & \\
\hline Hypersomnia events, $n$ & 1 & \\
\hline $\begin{array}{l}\text { Participants with } \geq 1 \text { sedative TEAE, } \\
n(\%)^{\mathrm{d}}\end{array}$ & $81(37.9)$ & \\
\hline Onset, days, median (range) & $21.0(1-702)$ & \\
\hline Duration, days, median (range) & $11.0(1-519)$ & \\
\hline \multicolumn{3}{|l|}{ Severity of event, $n(\%)^{\mathrm{e}}$} \\
\hline Mild & $81(64.8)$ & \\
\hline Moderate & $37(29.6)$ & \\
\hline Severe & $7(5.6)$ & \\
\hline $\begin{array}{l}\text { Events leading to discontinuation, } n \\
(\%)\end{array}$ & $2(1.6)$ & \\
\hline Unresolved sedative TEAEs, $n(\%)$ & $4(3.2)$ & \\
\hline
\end{tabular}

GXR guanfacine extended release, TEAE treatment-emergent adverse event

${ }^{a}$ Somnolence (2), dizziness (1), first-degree atrioventricular block (1), increased weight (1), aggression (1), drug abuse (1)

${ }^{\mathrm{b}}$ Stomatitis (2), concussion (2), appendicitis (1), gastroenteritis (1), lower limb fracture (1), post-procedural haemorrhage (1), radius fracture (1), upper limb fracture (1), wrist fracture (1), aggression (1), testicular torsion (1)

${ }^{c}$ Defined as somnolence, sedation, and hypersomnia

${ }^{\mathrm{d}}$ Percentage is calculated using the total number in the safety population as the denominator

${ }^{\text {e}}$ Percentage is calculated using the total number of sedative TEAEs as the denominator resolved spontaneously, with the duration of events ranging from 8 days to 176 days (duration was not reported for one resolved event). The unresolved aggression TEAE occurred in the participant reported to abuse cannabis, who was withdrawn from the study; this TEAE was not considered by the investigator to be related to GXR.

\section{Vital signs and ECG parameters}

Following an initial decrease, pulse rate stabilized during dose maintenance and returned to baseline levels during dose tapering (Fig. 3a). Mean change in pulse rate from baseline to the final assessment was $-5.5 \mathrm{bpm}$ (SD, 12.98). PCI events of low and high supine pulse rates $(\leq 50 ; \geq 100 \mathrm{bpm})$ were reported in 13.2 and $6.6 \%$ of participants, respectively (Supplementary Table 2). The events of low supine pulse rate were generally more common during dose optimization (weeks 3-8), whereas those of high supine pulse rate were most frequently reported at baseline and during off-treatment follow-up. Of the 28 individuals with PCI low supine pulse rate, $9(32.1 \%)$ also reported TEAEs of headache.

The mean supine SBP and DBP decreased after initiation of GXR therapy, reached a minimum after approximately 4 weeks, gradually returned to baseline levels during the second year of treatment, and increased further during dose tapering (Fig. 3b). Mean changes in SBP and DBP from baseline to the final assessment were $0.6 \mathrm{mmHg}$ (SD, 9.32) and $0.2 \mathrm{mmHg}(\mathrm{SD}, 9.17)$, respectively. PCI events of low SBP and low DBP were more common in adolescents than in children and their frequencies peaked at weeks 3 and 4 .

Changes in ECG parameters from baseline to the final assessment included a decrease in mean heart rate of - 7.6 bpm (SD, 13.76), an increase in mean PR interval of $+3.4 \mathrm{~ms}(\mathrm{SD}, 12.48)$ and an increase in mean QTcF interval of $+0.7 \mathrm{~ms}$ (SD, 16.01). The proportion of participants with a PCI ECG result was below 6\% for all ECG parameters except for low heart rate (11.8\% of participants). No participant had a PCI QTcF interval while on treatment.

PCI vital sign results by weight-adjusted dose are summarized in Supplementary Table 3.

\section{Height, weight and body mass index}

Mean $z$-scores for height, weight and BMI remained stable throughout the GXR treatment period. Of note, mean $z$-scores for weight and BMI were greater than zero at baseline (Fig. 4).

Most participants (163/207) remained in their baseline BMI percentile category at the final assessment, 17/207 shifted down a category and 27/207 shifted up (Supplementary Table 4). Of the 157 participants categorized as having a healthy weight (BMI $\geq 5$ th to $<85$ th percentiles) at baseline, the majority (137) remained in the healthy weight category 


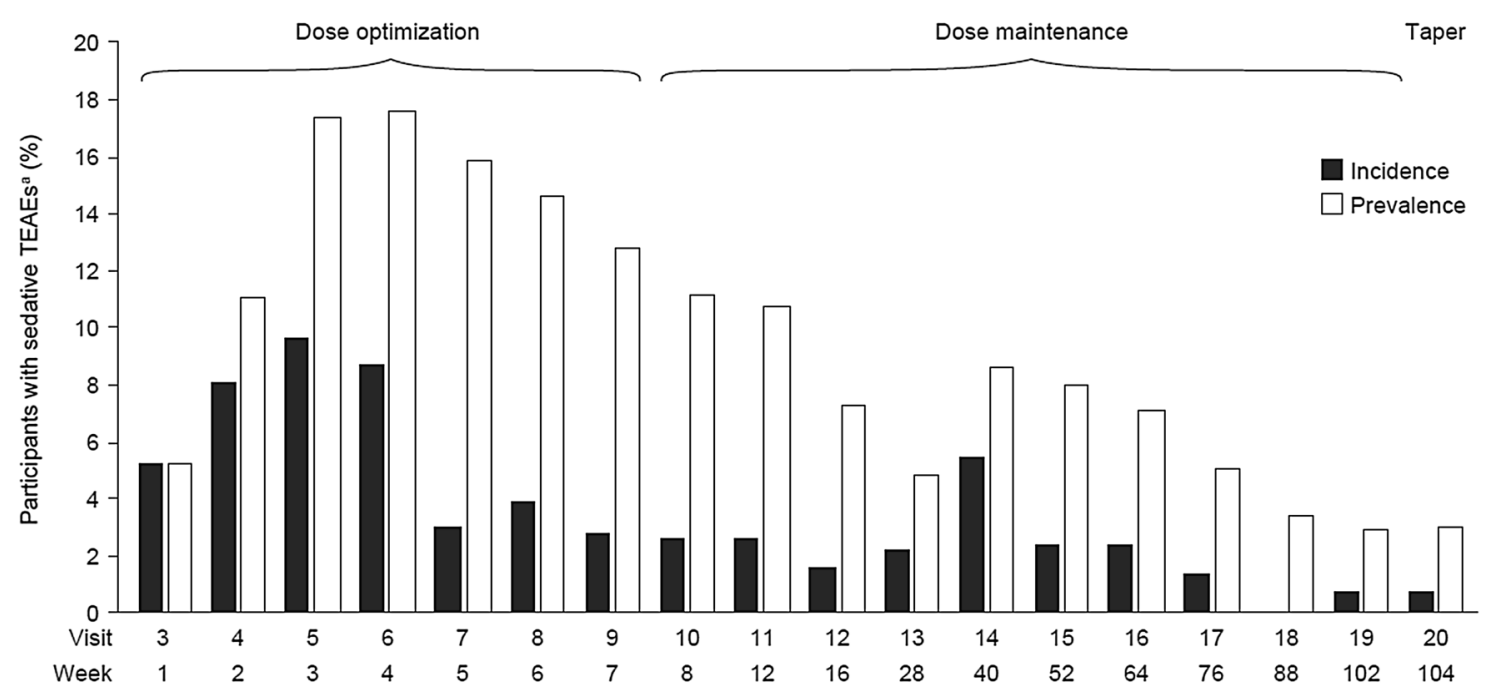

${ }^{a}$ Defined as somnolence, sedation and hypersomnia.

TEAE, treatment-emergent adverse event.

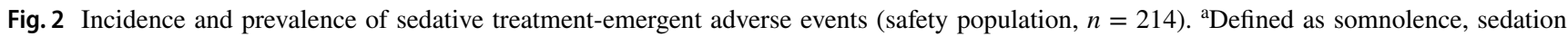
and hypersomnia. TEAE treatment-emergent adverse event

at the final assessment, five shifted to being underweight (BMI $<5$ th percentile) and 15 shifted to a higher weight category (14 became overweight [BMI $\geq 85$ th to $<95$ th percentiles] and one became obese [BMI $\geq 95$ th percentile]). All participants who shifted to a higher weight category from the healthy weight category had a BMI above the 50th percentile at baseline. Of the 45 participants in the overweight category at baseline, approximately half (23) remained overweight and approximately equal numbers shifted either down or up a category (12 shifted to the healthy weight category and 10 shifted to the obese category). All 14 participants in the obese category at the final assessment had a BMI above the 80th percentile at baseline (1 healthy weight, 10 overweight, 3 obese).

One participant had a TEAE of increased weight that led to early termination and was considered to be related to GXR. The participant (an 8-year-old boy) was overweight at baseline (BMI $18.63 \mathrm{~kg} / \mathrm{m}^{2}$ ) and obese (BMI $23.9 \mathrm{~kg} / \mathrm{m}^{2}$ ) on day 506, which led to him withdrawing from the study. He was still obese at follow-up (day 527; BMI $23.6 \mathrm{~kg} / \mathrm{m}^{2}$ ) and the TEAE was considered to be resolved on day 792 (no height, weight or BMI data reported).

\section{Columbia-Suicide Severity Rating Scale}

Overall, five participants responded 'yes' while on treatment to one or more suicidal ideation categories of the C-SSRS; four indicated a 'wish to be dead' and two reported 'nonspecific active suicidal thoughts'. One adolescent reported an event of non-suicidal self-injurious behaviour. No participants reported active suicidal ideation and there were no incidences of suicidal behaviour, including attempted or completed suicide. Additionally, one child who withdrew from the study responded 'yes' to the suicidal ideation category of 'wish to be dead' while not on treatment at his early termination visit after his last dose (the reason given for early termination was 'withdrawal by participant').

\section{Efficacy analyses}

Assessment of ADHD symptoms was a secondary objective of SPD503-318. At the final assessment, there was a significant mean change in ADHD-RS-IV total score from baseline of -19.8 (standard error of the mean [SEM], 0.84; nominal $p<0.0001$ ) (Supplementary Fig. 2a). Much of the improvement occurred during weeks 1-4 (mean change from baseline at week 4, - 14.9 [SEM, 0.66]) and scores continued to decrease throughout the study. Mean changes from baseline to the final assessment were also significant for both the ADHD-RS-IV Hyperactivity/Impulsivity subscale score $(-10.1$ [SEM, 0.44; nominal $p<0.0001])$ and Inattentiveness subscale score ( -9.8 [SEM, 0.47 ; nominal $p<0.0001]$ ).

Reductions in CGI-S scores (indicating improvement) were also observed over the 2 -year treatment period. The proportion of participants considered to be normal/borderline mentally ill improved from 1.0\% (2/208) at baseline 
Fig. 3 Mean a supine pulse rate, b supine systolic blood pressure and supine diastolic blood pressure (safety population, $n=214)$. Error bars show the standard error of the mean. $B L$ baseline, $F A$ final assessment, $F U$ follow-up
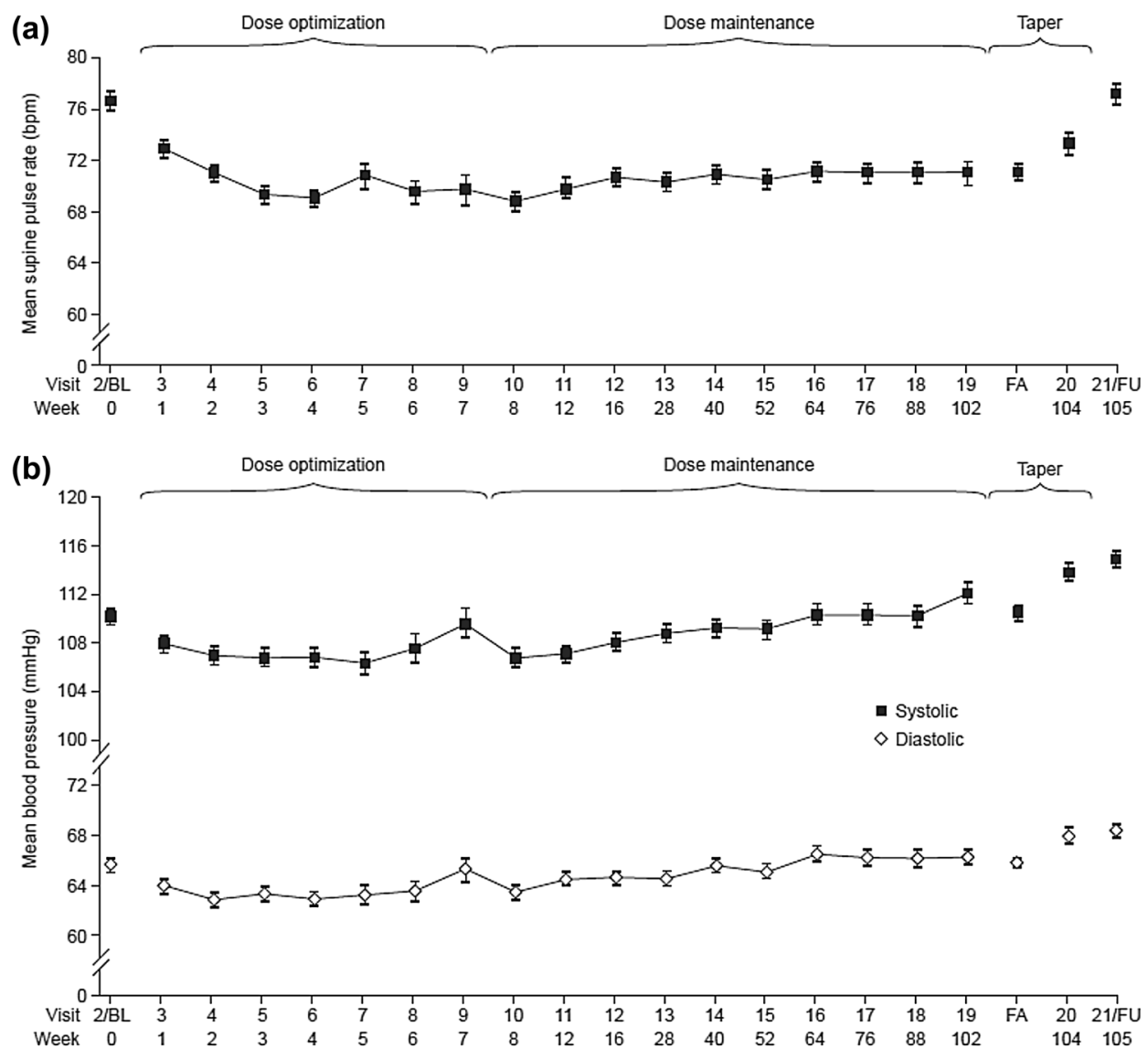

Error bars show standard error of the mean.

$\mathrm{BL}$, baseline; FA, final assessment; FU, follow-up. to $46.4 \%(96 / 207)$ at the final assessment (Supplementary Fig. 2b).

\section{Discussion}

SPD503-318 is the first 2-year trial to assess the safety of GXR at doses of up to $7 \mathrm{mg}$ /day in children and adolescents with ADHD, thereby extending the experience gained from the previous long-term studies in which GXR was used at doses of up to $4 \mathrm{mg} / \mathrm{day}$. SPD503-318 was designed to provide more information on known safety-related effects of GXR, including sedation, cardiovascular outcomes, growth, and weight. The frequency and nature of TEAEs reported in this large study involving over 200 participants were consistent with the previously reported safety profile of GXR in short- and long-term studies [15-18, 20, 21]. No new safety signals were identified.

Over $60 \%$ of participants completed the 2-year study; in contrast, the previous long-term studies of GXR were completed by 17.5 and $22.9 \%$ of participants only [20, 21].
The reason for this discrepancy is not clear. In the present study, rates of discontinuation due to 'withdrawal of consent' (17\%) and 'lost to follow-up' (2\%) were notably lower than in the two earlier studies ( $28 \%$ and $14-15 \%$, respectively) $[20,21]$. As long-term, open-label, dose-optimized, extension trials of GXR in children and adolescents, all three studies were of similar design, although the present study permitted a higher maximum dose of GXR ( $7 \mathrm{mg} /$ day versus $4 \mathrm{mg} /$ day) and enrolled a higher proportion of adolescents (39 versus $\leq 26 \%$ ) than earlier studies [20, 21]. In the present study, despite the higher permitted maximum dose, the proportion of participants withdrawing because of lack of efficacy (about 9\%) was similar to that reported in both the previous long-term studies (about 10\%) [20, 21].

There was no consistent pattern of increasing incidence of TEAEs with increasing dose, probably reflecting that this was a dose-optimized study. The majority of TEAEs reported here were of mild or moderate severity. TEAEs led to early termination in $3.3 \%$ of participants. The antecedent trials were discontinued by $20.9 \%$ (SPD503-316) and $51.6 \%$ (SPD503-315, randomized-withdrawal phase) of 

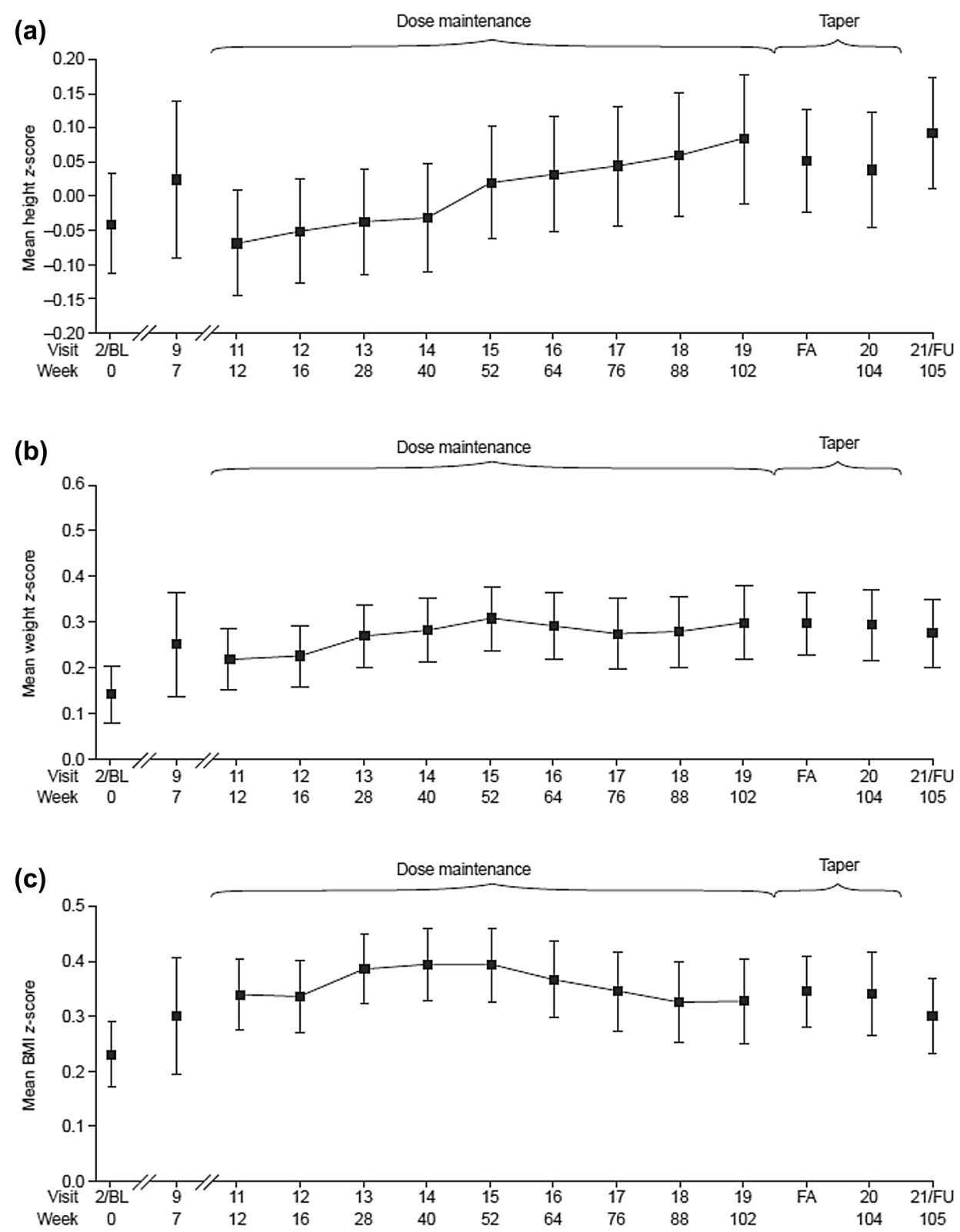

Error bars show standard error of the mean.

Height and weight were assessed at BL, week 7 and weeks 12-105. Height, weight and BMI $z$-scores adjusted for age and sex were calculated according to the 2000 Centers for Disease Control and Prevention growth charts for children and adolescents aged $2-<20$ years.

BL, baseline; BMI, body mass index; FA, final assessment; FU, follow-up.

Fig. 4 a Height, b weight, and c BMI $z$-scores (safety population, $n=214$ ). Error bars show the standard error of the mean. Height and weight were assessed at BL, week 7 and weeks 12-105. Height, weight, and BMI $z$-scores adjusted for age and sex were calculated

participants receiving GXR. Of these, 7.8 and $1.9 \%$ of participants, respectively, withdrew because of adverse events $[17,19]$. In line with the previous short- and long-term according to the 2000 Centers for Disease Control and Prevention growth charts for children and adolescents aged $2-<20$ years. $B L$ baseline, $B M I$ body mass index, $F A$ final assessment, $F U$ follow-up

studies, the most frequently reported TEAEs were somnolence, headache, and fatigue [15-18, 20,21]. The potential sedative effects of GXR are of particular interest; in the 
present study, $37.9 \%$ of participants experienced at least one sedative TEAE. In addition, $20.1 \%$ of participants reported fatigue, not included in the pre-defined grouping of sedative TEAEs in SPD503-318. Few of the sedative TEAEs led to early termination and none were considered serious. As in the previous long-term studies, sedative TEAEs were most frequently reported during the first month of GXR treatment and resolved spontaneously as treatment continued [20,21]. Based on observations from short-term trials, the incidence of sedative TEAEs appears to peak during the first week in which the target dose of GXR is reached [15, 16, 18], suggesting that the timing of the onset of sedative TEAEs may be linked to dose increases. In contrast, clinical improvement continued throughout the study, suggesting that symptomatic response and sedative TEAEs were not directly linked over time.

In the previous long-term GXR studies, dose-dependent decreases in blood pressure and heart rate were observed during early treatment [20,21]. Similarly, in the present study, there was a general trend for mean pulse rate and blood pressure to decrease during the first weeks of treatment, but then stabilize during the dose-maintenance period. Observed decreases in pulse rate and blood pressure were generally modest, asymptomatic and consistent with the known pharmacological effects of guanfacine [12, 14]. These parameters returned to baseline values during the second year of treatment or after treatment discontinuation. The overall prevalence of PCI events of low pulse rate or low blood pressure ranged from $13.2 \%$ (low pulse rate) to $52.4 \%$ (low DBP in adolescents). The lack of data providing context for these PCI events, such as time of day and time since dose, limits interpretation of these results. However, there was no evidence to show that these participants were symptomatic. The proportion of participants with PCI low pulse rate who also reported TEAEs of headache was similar to that of the overall safety population (32.1 and $28.5 \%$, respectively). The observed increases in mean blood pressure during dose tapering suggest that vital signs should be monitored when discontinuing treatment. Of note, the magnitude of the mean increases in QTcF was consistent with the known effects of GXR and there were no PCI events of increased QTcF.

Height, weight, and BMI were stable throughout treatment. Most of those within the healthy BMI category at baseline remained in that category throughout the 2-year study, although a small number of individuals shifted to higher or lower BMI percentile categories. Most (13/14) of those who were in the obese category at the final assessment were overweight or obese at baseline. These findings highlight the importance of regular height and weight monitoring during GXR treatment, especially for those who are overweight before starting therapy. Of note, mean weight and BMI $z$-scores were greater than zero at baseline. However, the $z$-scores used CDC norms that were generated from data pre-dating 2000 and thus may not be representative of the current population [23].

While it would be unethical to carry out a 2-year trial with a placebo arm, the lack of placebo is an important limitation of SPD503-318, which restricts interpretation regarding the observed safety and efficacy signals. The openlabel design, resulting in a greater risk of bias than a randomized and blinded study, should also be taken into consideration. As should the LOCF imputation method, which is recognized as problematic because of the assumption that responses remain stable from the time of drug discontinuation, potentially leading to an over- or under-estimation of the real response rate. LOCF also assumes that the probability of discontinuation is not related to side effects or change in symptoms. However, as described above, the present study had a relatively high completion rate with a low proportion of discontinuations because of lack of efficacy or TEAEs. Furthermore, the maintenance of efficacy of GXR has been demonstrated previously in a randomized, placebocontrolled withdrawal study [19]. In addition, clinical symptoms appeared to improve throughout the study to a similar magnitude to that observed in the previous long-term studies $[20,21]$, with a mean ADHD-RS-IV total score at the final assessment of 16.8. Further studies, including independent non-industry sponsored trials, will help to validate the findings reported here.

Finally, participants in this study were selected according to various inclusion and exclusion criteria, which may have generated a population not truly reflective of clinical practice. Although participants with a high level of co-morbid symptoms could be included in this study, children and adolescents with co-morbid disorders other than ODD were excluded. The subgroup of participants with ODD was too small (less than $10 \%$ of the overall study population) to permit a robust subgroup analysis of GXR in individuals with ADHD and ODD.

\section{Conclusions}

This 2-year European extension study confirms that GXR at doses of up to $7 \mathrm{mg} /$ day is well tolerated during longterm use, with a safety profile similar to that observed in the previous, short-term international trials and long-term US studies of GXR. Height, weight, and BMI were stable throughout treatment. Initial reductions in pulse rate and blood pressure trended towards baseline and stabilized during the second year of treatment. Overall symptom response was consistent with results from the previous studies, with a large proportion of participants remaining on treatment throughout the study. 
Acknowledgements We thank the patients and investigators who took part in this study. Under the direction of the authors and funded by Shire International GmbH, Dr. AL Jones and Dr. I Kaufmann of Oxford PharmaGenesis, Oxford, UK, provided writing assistance for this publication. Editorial assistance in formatting, proofreading, copy-editing, and fact-checking was also provided by Oxford PharmaGenesis. Dr. T Werner-Kiechle from Shire International GmbH reviewed and edited the manuscript for scientific accuracy. Although employees of the sponsor were involved in the design, collection, analysis, interpretation, and fact-checking of information, the content of this manuscript, the interpretation of the data, and the decision to submit the manuscript for publication in European Child and Adolescent Psychiatry was made by the authors independently. Dr. J Gu served as the statistical expert for this research.

\section{Compliance with ethical standards}

Conflict of interest J Gu and B Robertson are employees of Shire and own stock or stock options. B Dirks is a former employee of Shire. The following authors have received compensation for serving as consultants or speakers for, or they or the institutions they work for have received research support or royalties from, the companies or organizations indicated: M Huss (Actelion, Eli Lilly, Engelhard Arzneimittel, Janssen-Cilag, Medice, Novartis, Shire, and Steiner Arzneimittel); J Newcorn (Akili Interactive, Alcobra, Enzymotec, Ironshore, Lundbeck, Medice, National Football League, Neos Therapeutics, NLS, Pearson, Shire, Sunovion, and Supernus); and JA Ramos-Quiroga (Ferrer, Eli Lilly, Janssen-Cilag, Novartis, Lundbeck, Shire, Rovi, and Laboratorios Rubió). Study funded by Shire Development LLC.

Open Access This article is distributed under the terms of the Creative Commons Attribution 4.0 International License (http://creativeco mmons.org/licenses/by/4.0/), which permits unrestricted use, distribution, and reproduction in any medium, provided you give appropriate credit to the original author(s) and the source, provide a link to the Creative Commons license, and indicate if changes were made.

\section{References}

1. Polanczyk G, de Lima MS, Horta BL, Biederman J, Rohde LA (2007) The worldwide prevalence of ADHD: a systematic review and metaregression analysis. Am J Psychiatry 164(6):942-948. https://doi.org/10.1176/ajp.2007.164.6.942

2. Polanczyk GV, Salum GA, Sugaya LS, Caye A, Rohde LA (2015) Annual research review: a meta-analysis of the worldwide prevalence of mental disorders in children and adolescents. J Child Psychol Psychiatry 56(3):345-365. https://doi.org/10.1111/ jcpp. 12381

3. Barkley RA, Fischer M, Smallish L, Fletcher K (2002) The persistence of attention-deficit/hyperactivity disorder into young adulthood as a function of reporting source and definition of disorder. J Abnorm Psychol 111(2):279-289. https://doi. org/10.1037/0021-843X.111.2.279

4. Faraone SV, Biederman J, Mick E (2006) The age-dependent decline of attention deficit hyperactivity disorder: a meta-analysis of follow-up studies. Psychol Med 36(2):159-165. https://doi. org/10.1017/S003329170500471X

5. Arnold LE, Hodgkins P, Caci H, Kahle J, Young S (2015) Effect of treatment modality on long-term outcomes in attentiondeficit/hyperactivity disorder: a systematic review. PLoS One 10(2):e0116407. https://doi.org/10.1371/journal.pone.0116407
6. The British Psychological Society and The Royal College of Psychiatrists (2016) The NICE guideline on diagnosis and management of ADHD in children, young people and adults. National Institute for Health and Care Excellence, London

7. Wolraich M, Brown L, Brown RT, DuPaul G, Earls M, Feldman HM, Ganiats TG, Kaplanek B, Meyer B, Perrin J, Pierce K, Reiff M, Stein MT, Visser S, Subcommittee on attentiondeficit/hyperactivity disorder, steering committee on quality improvement and management (2011) ADHD: clinical practice guideline for the diagnosis, evaluation, and treatment of attention-deficit/hyperactivity disorder in children and adolescents. Pediatrics 128(5):1007-1022. https://doi.org/10.1542/ peds.2011-2654

8. Beau-Lejdstrom R, Douglas I, Evans SJ, Smeeth L (2016) Latest trends in ADHD drug prescribing patterns in children in the UK: prevalence, incidence and persistence. BMJ Open 6(6):e010508. https://doi.org/10.1136/bmjopen-2015-010508

9. Lachaine J, Beauchemin C, Sasane R, Hodgkins PS (2012) Treatment patterns, adherence, and persistence in ADHD: a Canadian perspective. Postgrad Med 124(3):139-148. https://doi. org/10.3810/pgm.2012.05.2557

10. Charach A, Figueroa M, Chen S, Ickowicz A, Schachar R (2006) Stimulant treatment over 5 years: effects on growth. J Am Acad Child Adolesc Psychiatry 45(4):415-421. https://doi. org/10.1097/01.chi.0000199026.91699.20

11. Swanson JM, Elliott GR, Greenhill LL, Wigal T, Arnold LE, Vitiello B, Hechtman L, Epstein JN, Pelham WE, Abikoff HB, Newcorn JH, Molina BS, Hinshaw SP, Wells KC, Hoza B, Jensen PS, Gibbons RD, Hur K, Stehli A, Davies M, March JS, Conners CK, Caron M, Volkow ND (2007) Effects of stimulant medication on growth rates across 3 years in the MTA follow-up. J Am Acad Child Adolesc Psychiatry 46(8):1015-1027. https://doi. org/10.1097/chi.0b013e3180686d7e

12. European Medicines Agency. Intuniv: EPAR - product information (2015). http://www.ema.europa.eu/docs/en_GB/document_ library/EPAR___Product_Information/human/003759/WC500 195130.pdf. Accessed 21 December 2016

13. Vannucci A, Nelson EE, Bongiorno DM, Pine DS, Yanovski JA, Tanofsky-Kraff M (2015) Behavioral and neurodevelopmental precursors to binge-type eating disorders: support for the role of negative valence systems. Psychol Med 45(14):2921-2936. https ://doi.org/10.1017/s003329171500104x

14. Intuniv ${ }^{\circledR}$ (guanfacine) capsules US prescribing information (revised March 2016). http://pi.shirecontent.com/PI/PDFs/Intun iv_USA_ENG.pdf. Accessed 14 October 2016

15. Biederman J, Melmed RD, Patel A, McBurnett K, Konow J, Lyne A, Scherer N (2008) A randomized, double-blind, placebo-controlled study of guanfacine extended release in children and adolescents with attention-deficit/hyperactivity disorder. Pediatrics 121(1):e73-e84. https://doi.org/10.1542/peds.2006-3695

16. Sallee FR, McGough J, Wigal T, Donahue J, Lyne A, Biederman J, SPD503 Study Group (2009) Guanfacine extended release in children and adolescents with attention-deficit/hyperactivity disorder: a placebo-controlled trial. J Am Acad Child Adolesc Psychiatry 48(2):155-165. https://doi.org/10.1097/chi.0b013e3181 $91769 \mathrm{e}$

17. Hervas A, Huss M, Johnson M, McNicholas F, van Stralen J, Sreckovic S, Lyne A, Bloomfield R, Sikirica V, Robertson B (2014) Efficacy and safety of extended-release guanfacine hydrochloride in children and adolescents with attention-deficit/ hyperactivity disorder: a randomized, controlled, phase III trial. Eur Neuropsychopharmacol 24(12):1861-1872. https://doi. org/10.1016/j.euroneuro.2014.09.014

18. Wilens TE, Robertson B, Sikirica V, Harper L, Young JL, Bloomfield R, Lyne A, Rynkowski G, Cutler AJ (2015) A randomized, placebo-controlled trial of guanfacine extended release 
in adolescents with attention-deficit/hyperactivity disorder. J Am Acad Child Adolesc Psychiatry 54(11):916-925. https://doi. org/10.1016/j.jaac.2015.08.016 (e912)

19. Newcorn JH, Harpin V, Huss M, Lyne A, Sikirica V, Johnson M, Ramos-Quiroga JA, van Stralen J, Dutray B, Sreckovic S, Bloomfield R, Robertson B (2016) Extended-release guanfacine hydrochloride in 6-17-year olds with ADHD: a randomised-withdrawal maintenance of efficacy study. J Child Psychol Psychiatry 57(6):717-728. https://doi.org/10.1111/jcpp.12492

20. Biederman J, Melmed RD, Patel A, McBurnett K, Donahue J, Lyne A (2008) Long-term, open-label extension study of guanfacine extended release in children and adolescents with ADHD. CNS Spectr 13(12):1047-1055. https://doi.org/10.1017/S1092 852900017107

21. Sallee FR, Lyne A, Wigal T, McGough JJ (2009) Long-term safety and efficacy of guanfacine extended release in children and adolescents with attention-deficit/hyperactivity disorder. J Child Adolesc Psychopharmacol 19(3):215-226. https://doi.org/10.1089/ cap. 2008.0080
22. European Medicines Agency. Summary of the risk management plan (RMP) for Intuniv (guanfacine) (2015). http://www.ema. europa.eu/docs/en_GB/document_library/EPAR_-_Risk-manag ement-plan_summary/human/003759/WC500190181.pdf. Accessed 21 December 2016

23. Kuczmarski RJ, Ogden CL, Guo SS, Grummer-Strawn LM, Flegal KM, Mei Z, Wei R, Curtin LR, Roche AF, Johnson CL (2002) 2000 CDC Growth Charts for the United States: methods and development. Vital Health Stat 11(246):1-190

24. DuPaul GJ (1998) ADHD Rating Scale-IV: checklists, norms, and clinical interpretation. Guilford Press, New York

25. Guy W (1976) Clinical global impressions. The ECDEU assessment manual for psychopharmacology-revised volume, 338th edn. US Dept. of Health, Education, and Welfare, Public Health Service, Alcohol, Drug Abuse, and Mental Health Administration, National Institute of Mental Health, Psychopharmacology Research Branch, Division of Extramural Research Programs, Rockville, pp 218-222 